\title{
ARTROPLASTIA TOTAL DO QUADRIL E A HIDROTERAPIA COMO MÉTODO DE REABILITAÇÃO
}

\section{TOTAL HYPOPLASTY ARTHROPLASTY AND HYDROTHERAPY AS A REHABILITATION METHOD}

\author{
Edvânia de Alencar Martins Duarte \\ Acadêmica do $9^{\circ}$ Período do Curso de Fisioterapia UNIPAC. Brasil. \\ edvaniaduarte13@hotmail.com
}

Matteus Cordeiro de Sá

Especialista em Fisioterapia Traumato-Ortopédica -UNIPAC. Curso de Fisioterapia matteuscordeirodesa@gmail.com

\section{Resumo}

Quando a articulação do quadril estiver degenerada a ponto das atividades de vida diária, especialmente andar, tornarem-se muito dolorosa, faz-se necessário à substituição do quadril, chamada de Artroplastia Total de Quadril (ATQ). A fisioterapia no pós-operatório, através de seus vastos recursos científicos, tem como objetivo promover e reabilitar a saúde desses pacientes, utilizando técnicas especializadas para adequada aplicação de recursos (físicos e naturais). A hidroterapia utiliza o meio aquático para alcançar objetivos fisioterapêuticos: manutenção ou ganho de amplitude de movimento, força muscular, analgesia, promoção de independência funcional. Torna-se indispensável à intervenção fisioterapêutica imediatamente no pós-operatório, com o objetivo de validar a reabilitação. Sob esse viés, o presente trabalho visou analisar os resultados e benefícios obtidos através da hidroterapia na ATQ, com o objetivo de verificar o efeito do tratamento sobre a força muscular, propriocepção e equilíbrio no período pós-operatório. Observou-se também a funcionalidade da marcha do paciente submetido ao procedimento cirúrgico no processo de reabilitação, discorrendo sobre a eficácia dos exercícios hidroterapêuticos no pós-operatório de ATQ, através de pesquisa bibliográfica. O aprofundamento dessa discussão, em diálogo com os autores citados, permite a conclusão de que houve melhoria do quadro clínico nos pacientes que realizaram fisioterapia no pós-operatório de ATQ, demonstrando a importância do tratamento precoce na recuperação da funcionalidade e qualidade de vida do paciente.

Palavras-chaves: Artroplastia Total de Quadril; Reabilitação Fisioterapêutica; Hidroterapia. 
Abstract

When the hip joint is degenerated to the point where activities of daily living, especially walking, become very painful, it is necessary to replace the hip called Total Hip Arthroplasty (HCA). Postoperative physiotherapy, through its vast scientific resources, aims to promote and rehabilitate the health of these patients, using specialized techniques for adequate application of resources (physical and natural). Hydrotherapy uses the aquatic environment to achieve physiotherapeutic goals: maintenance or gain of range of motion, muscular strength, analgesia, promotion of functional independence. It becomes indispensable to the physiotherapeutic intervention immediately in the postoperative period, with the purpose of validating the rehabilitation. Under this bias, the present study aimed to analyze the results and benefits obtained through hydrotherapy in ATQ, in order to verify the effect of treatment on muscle strength, proprioception and balance in the postoperative period. It was also observed the functional gait of the patient undergoing the surgical procedure in the rehabilitation process, discussing the efficacy of the hydrotherapeutic exercises in the postoperative period of TKA, through bibliographic research. The deepening of this discussion, in dialogue with the aforementioned authors, allows the conclusion that there was an improvement in the clinical picture in the patients who underwent physiotherapy in the postoperative period of HCA, demonstrating the importance of early treatment in the recovery of the patient's functionality and quality of life.

Keywords: Total Hip Arthroplasty; Physiotherapeutic Rehabilitation; Hydrotherapy.

\section{Introdução}

Entende-se por artroplastia total de quadril um método cirúrgico usado no tratamento de acometimentos da articulação coxofemoral, em que acontece a substituição total da articulação natural por outra artificial, com um implante protético (feito de materiais não orgânicos), (PEREIRA et al., 2014). Esse tratamento será adequado para pacientes por meio de exames físicos que mostrarem: incapacidade de andar por um percurso sem ter que parar, impedimento para efetuar suas tarefas de vida diária, dificuldade de subir escadas, com amplitude de movimento limitada e dor ao movimentar-se, (SKINNER; Mc MAHON, 2015).

Depois de submeterem-se à cirurgia de ATQ, a maior parte dos pacientes ainda manifestam restrição de movimento, hipotrofia muscular significativa, dor, fraqueza do membro afetado e alterações na marcha, seguindo de uma diminuição dos níveis de movimento, independência e atividade física, (GALEA et al., 2008; WAGENMAKERS et al., 2008).

A hidroterapia promove diferentes efeitos e reações, daqueles destacados em solo, contendo a melhoria da circulação periférica, favorecimento do retorno venoso, além de causar um resultado massageador e relaxante. Os exercícios desempenhados em água 
aquecida são bastante bem aceitos, pois o ambiente morno auxilia a cessar ou diminuir a dor e espasmos musculares. É possível adquirir uma agradável força através dos movimentos nos exercícios feitos na água e, ainda, a oportunidade de treinamento em diversas velocidades. Esses elementos fazem com que o exercício aquático seja um método para acréscimo de resistência e força muscular, (FERREIRA et al., 2008).

A hidroterapia é uma alternativa considerável para a reabilitação e mudanças funcionais, pois dispõe de princípios físicos, fisiológicos e cinesiológicos alcançados por meio da imersão do corpo em piscina aquecida. As particularidades físicas e o aquecimento da água exercem um considerável papel no avanço e a preservação da amplitude de movimento (ADM) das articulações, do relaxamento e na diminuição da tensão muscular, (CANDELORO e CAROMANO, 2007).

O trabalho situa-se na necessidade de mostrar a atuação do fisioterapeuta no pósoperatório de cirurgia de ATQ. Desta forma, detectar o método cirúrgico, o perfil do paciente e o que envolve esse momento auxilia o fisioterapeuta a prever e a prover os cuidados que sejam capazes de evitar problemas e estar alerta para atender determinadas situações previstas ou inusitadas, não apenas no pós-operatório, mas também nos outros períodos operatórios.

A presente pesquisa trata-se de um artigo apresentado ao Curso de Fisioterapia da Universidade Presidente Antônio Carlos (UNIPAC), e o objetivo geral deste estudo é analisar o efeito do tratamento hidroterapêutico na reabilitação pós-operatória de pacientes que realizaram a ATQ, que é um assunto de grande relevância, pois o tema é repleto de peculiaridades e pormenores que necessitam de pesquisas e comparações, a fim de que possa ser aplicada de forma a cumprir sua finalidade.

Este estudo foi elaborado a partir da realização de uma revisão literária referente aos efeitos da hidroterapia na reabilitação de pacientes com artroplastia total do quadril. O estudo foi feito através da pesquisa em capítulos de livros, artigos científicos, revistas especializadas e bases eletrônicas de dados como SCIELO (Scientific Electronic Library Online), LILACS (Literatura Latino Americana e do Caribe em Ciências da Saúde) e Google Acadêmico. Foram selecionados alguns artigos, utilizando o cruzamento entre os descritores: anatomia do quadril, artroplastia total do quadril e hidroterapia. Foram avaliados apenas os artigos que corresponderam aos interesses do estudo. Os artigos foram selecionados através do método de 
consulta, leitura e avaliação do conteúdo com o objetivo de identificar os benefícios da hidroterapia na reabilitação de pacientes com artroplastia total do quadril. A coleta de dados foi do ano de 2005 a 2018, exceto autores que são referência, exemplo: Bates e Hanson 1993, Campion 2000. Foram utilizados artigos na linguagem portuguesa e inglesa.

\section{Anatomia do quadril}

Segundo Kisner e Colby (2016), o quadril é uma articulação do tipo bola e soquete (esferoide) triaxial formada pela cabeça do fêmur e pelo acetábulo da pelve. É amparado por uma cápsula articular forte que é sustentada pelos ligamentos pubofemoral, iliofemoral e isquiofemoral. As duas articulações do quadril vão se unir por meio da pelve óssea e da coluna vertebral nas articulações lombossacrais e sacroilíaca. (vide figura 1).

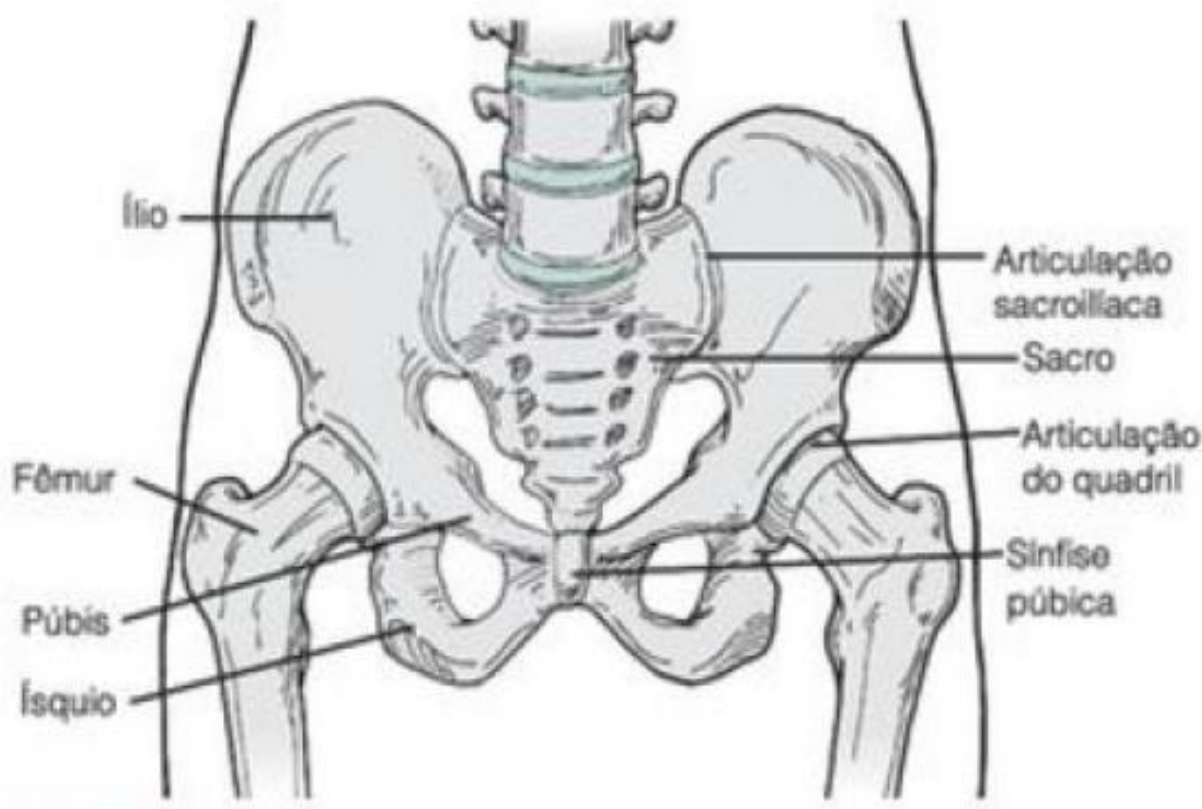

Fonte: Kisner e Colby 2016.

Figura 1: Ossos e articulações da pelve e do quadril

A articulação coxofemoral é composta pela articulação do fêmur com o acetábulo que se localiza na pelve, sendo a pelve composta pelos ossos chamados ílio, ísquio e púbis, (PRENTICE, 2012). 
Existe três ossos no quadril conhecidos por: ílio, ísquio e púbis, que se articulam na parte posterior com os ossos do sacro constituindo a articulação sacro-ilíaca, anteriormente na sínfise púbica e lateralmente com o osso do fêmur na articulação coxofemoral, (TORTORA; DERRICKSON, 2015).

O fêmur apresenta uma cabeça esférica que se encaixa em um soquete profundo conhecido acetábulo localizado na pelve, coberto por uma massa de tecido adiposo, cápsula e ligamentos, (PRENTICE, 2012). O fêmur é o maior osso e mais forte do corpo e sua extremidade proximal contém uma cabeça recoberta por uma delicada camada de cartilagem, um colo e um trocânter maior e outro menor, (DUTTON, 2010). A cabeça do fêmur com sua forma convexa, durante o movimento da coxa desliza no sentido oposto da mesma, (LIPPERT, 2013).

O acetábulo é uma superfície côncava que recebe a cabeça do fêmur, que tem seu aspecto convexa constituindo a articulação coxofemoral do tipo esferoide, (CAMARGO JR; DIONÍSIO, 2016), ele é composto pela fusão dos três ossos que formam a pelve (ílio, ísquio e púbis), (DUTTON, 2010).

Encontra-se uma estrutura que amplia a estabilidade articular entre o osso do quadril e o fêmur, chamada de lábio, lábrum ou borda acetabular (e que reveste o acetábulo), esta estrutura aprofunda o acetábulo deixando que assim aconteça o aumento da estabilidade articular entre o osso do quadril e o fêmur, gerando uma pressão intra-articular negativa na articulação do quadril, (DUTTON, 2010; CAMARGO JR; DIONÍSIO, 2016).

O quadril tem como finalidade inicial no decorrer da postura ereta estática e durante atividades dinâmicas suportar o peso da cabeça, tronco e braços, além de propiciar transmissão de forças entre pelve e extremidades inferiores, (DUTTON, 2006).

É uma articulação bastante estável e correspondente a isso sua amplitude de movimento é menor, se comparada ao movimento de outras articulações como a do ombro, (LIPPERT, 2013). Essa articulação tem como movimentos: flexão-extensão, abdução-adução e rotação lateral-medial, (MORAES, 2005).

\section{Artroplastia total do quadril}


Entende-se por artroplastia total do quadril (ATQ), como uma pratica cirúrgica de reconstrução protética do quadril, (SILVA, 2014), usada em pacientes com lesão rigorosa da articulação coxofemoral, como fraturas de colo femoral, necrose vascular da cabeça do fêmur, displasia do desenvolvimento do quadril, (NUNES, 2010). Esse método de modificação ou troca da articulação é utilizada quando o grau de dor que este indivíduo sente já se aponta como um aspecto limitante nas suas atividades de vida diária, e as condutas conservadoras não forem efetivas em proporcionar uma evolução da função do membro acometido, (MORAES, 2005).

Esse método de substituição da articulação do quadril surgiu com Jonh Charnley, um cirurgião da Inglaterra, e foi descrito por Herbert e colaboradores em 2009, em que o trabalho de Charnley, iniciou em 1958 com o desenvolvimento da prótese, composta de um componente femoral metálico e do componente acetabular plástico, (SCHWARTSMANN, 2012; KISNER; COLBY, 2009).

Três fatores críticos são fundamentais para que ocorra sucesso na artroplastia total de quadril: escolha do paciente, escolha da técnica cirúrgica e seleção do implante, (HERBERT, et al., 2009). Já para Didomenico e Paes (2010), deve se levar em conta a idade do paciente, a qualidade do osso e a experiência do cirurgião.

As próteses de quadril contêm vários modelos e design e visaram melhorar a qualidade de vida, reparar a estabilidade e função da articulação do quadril, além de recuperar os movimentos o mais próximo possível da biomecânica normal, (NUNES, 2010).

A prótese do tipo cimentada é recomendada para pacientes de até 60 anos fisicamente ativos, pois este tipo de fixação evita que a prótese se folgue, (DIDOMENICO; PAES, 2010).

A artroplastia total de quadril é destinada em quadros álgicos intensos no quadril, limitação do movimento e da sustentação do peso corporal, tendo como resultado função limitada, (GANN, 2005).

Em geral quanto mais intensivo o quadro álgico pré-operatório, maior a satisfação do paciente após artroplastia total de quadril, (BONVICINE, 2006).

A existência de infecção ativa na articulação é uma contraindicação específica, seja ela uma infecção ativa da articulação do quadril, pacientes sem massa óssea suficiente para fixação dos componentes, distúrbios neurológicos não controlados, infecção na bexiga, pacientes com osteoporose e osteopenia, (DIDOMENICO; PAES 2010). 
Conforme Nunes et al. (2010) e Matos (2006), a artroplastia total de quadril tem a função de aliviar a dor do paciente possibilitando melhor biomecânica para o movimento, reparar a função e a estabilidade da articulação assim como resgatar a funcionalidade aproximando-se da biomecânica normal proporcionando uma melhor qualidade de vida. Para Didomenico e Paes (2010), o principal objetivo é o alívio do quadro álgico.

\section{Hidroterapia}

A hidroterapia é recurso terapêutico antigo da fisioterapia, que vem ocupando espaço cada vez mais, ela utiliza dos efeitos cinesiológicos, físicos e fisiológicos, proveniente da imersão de uma parcial ou total do corpo no meio aquático. As características físicas da água desempenham uma ação importante para fins terapêuticos, que são dependentes da temperatura da água, duração e intensidade do tratamento. Seus efeitos são térmicos e mecânicos, e a soma desses, aos benefícios do tratamento em imersão trazem resultados fisiológicos maiores, melhorando todo o corpo, (VIEIRA et al, 2017).

A hidroterapia é sugerida para o tratamento de idosos, por mostrarem desordem que se tornam obstáculo para que os exercícios sejam realizados em solo, já no ambiente aquático terá uma diminuição do peso corporal, que favorecera um ambiente confortável, levando uma liberdade maior nos movimentos, resultando uma alternativa de prevenção e reabilitação segura para o paciente, (FACCI et al, 2007; VIERA et al, 2017).

A função da hidroterapia é a reabilitação de inúmeras patologias levando benefício terapêutico, fisiológico e físico, esses ganhos se dão por meio das propriedades oferecidas pela água, que são elas a flutuação, viscosidade, temperatura, turbulência e pressão hidrostática, (OLIVEIRA; SOUZA, 2018).

A imersão aquática possui propósitos fisiológicos relevantes que se abrangem sobre todos os sistemas e a homeostase. Estes resultados podem ser tanto imediatos quanto tardios, propiciando assim, que a água seja utilizada para fins terapêuticos em uma grande variedade de problemas orgânicos. A terapia aquática parece ser benéfica no tratamento de pacientes com distúrbios músculo-esqueléticos, neurológicos, cardiopulmonares, entre outros, (GIMENES et al, 2005). 
Entre as inúmeras técnicas de hidroterapia, destacam-se duas que apresentam melhores resultados a hidrocinesioterapia e o Bad Ragaz: A hidrocinesioterapia visa à reabilitação humana pelos princípios da aplicação de exercícios físicos juntamente com as propriedades da água, (MEEREIS, 2013). Alguns dos efeitos obtidos podem ser diminuição de espasmos e quadro álgico, aumento da circulação sanguínea, melhora na amplitude de movimento e fortalecimento muscular, (SILVA, 2012). A técnica Bad Ragaz, tem como função o auxílio na adequação do tônus muscular, amplitude de movimento articular, fortalecimento muscular, restauração de padrões normais de movimento, relaxamento, além de melhorar a resistência geral. O terapeuta permite estabilidade e a posição de suas mãos influência a movimentação do paciente. Os movimentos executados nesta técnica podem ser isométricos quando o paciente mantém uma posição fixa enquanto é movido sobre a água pelo terapeuta; isotônico no momento em que o paciente executa a ação, porém é desestabilizado pelo terapeuta e isocinético quando o terapeuta fornece fixação enquanto o paciente se move ativamente na água, (MENEGHETTI et al., 2009).

\section{Hidroterapia na artroplastia total do quadril}

Uma vez que já apresentado o assunto em questão, onde já foi passado sobre a AQT e seus sinais e sintomas, bem como as propriedades físicas da água e seus efeitos sobre o corpo imerso, torna-se importante trazer as ideias dos autores pesquisados citando-se os exercícios hidroterapêuticos, e ver suas relações entre se.

Entre as propriedades físicas da água que são utilizadas pela hidroterapia, a força de flutuação da água é de suma importância para a reabilitação, porque irá reduzir o peso corpóreo nesse ambiente, diminuindo assim o impacto sobre as articulações e o risco de lesões. O real efeito da deambulação e apoio precoce em pacientes com prótese de quadril é muito estudado, e em estudos semelhantes, observou que esses efeitos por meio de estudos radiológicos, concluindo que nenhuma alteração em longo prazo foi causada pelo apoio precoce. Entretanto, muitas vezes o apoio imediato não é bem suportado pelos pacientes, principalmente pelo receio em apoiar o membro operado, (STROM et al. 2007). 
Diante dessas circunstâncias o mesmo autor relata que a fisioterapia realizada no meio líquido oferece vantagens, pois com a diminuição da carga proporcionada pelo efeito de flutuação, o processo de reabilitação é facilitado e o paciente sentirá mais seguro para realizar movimentos e exercícios que envolvam o apoio do membro operado, (STROM et al. 2007).

Concordando com Strom et al. 2007, Carregaro em 2008 mostra que o progresso de descarga de peso na água é um feito fundamental para a reabilitação, na medida em que pode ser usado como avanço na produção dos exercícios tendo por base os níveis de profundidade na altura dos joelhos, quadril e pescoço, podem diminuir o peso em 15\%, $50 \%$ e $90 \%$, relativamente, (CARREGARO, 2008).

Campion (2000), relata alguns dos objetivos terapêuticos dos exercícios na água tais como: alívio dos espasmos musculares e da dor, aumento da amplitude de movimento nas articulações ou manutenção, fortalecimento muscular e maior flexibilidade aos exercícios. Há uma melhora significativa na circulação, no equilíbrio e na coordenação postural, além de assegurar incentivo nas atividades funcionais do paciente entre demais vantagens.

Soares (2014), afirma que pela ação de flutuação tem-se o auxílio na mobilidade das articulações rígidas, obtendo uma amplitude maior com um aumento mínimo de dor. Já em relação aos exercícios de fortalecimento com paciente submerso que estão estabelecidos nos conceitos físicos da hidrostática, irá gerar resistência em diversas direções frequentes aos movimentos. A dificuldade aumenta à medida que a força é exercida contra ela, o que leva há uma sobrecarga mínima nas articulações, facilitando que esse paciente tenha um melhor desempenho.

Segundo Rosa (2013), a hidroterapia tem indicação eficaz para o aperfeiçoamento do equilíbrio postural, no decorrer da realização de alguns movimentos podem se assemelhar a atividades práticas e também no decorrer da marcha. Sendo assim, esses resultados mostram a importância da hidroterapia no cuidado e melhora do equilíbrio postural em indivíduos com estabelecidas limitações.

A hidroterapia pode ser usada como auxilio essencial aos pacientes, trabalhando a flexibilidade muscular e articular, além de trabalhar a resistência muscular e aeróbica, limitando a dor, possibilitando uma melhora na qualidade de vida e na capacidade funcional dos pacientes, a hidroterapia pode ser utilizada de forma recreativa no paciente, (SOARES, 2014). 
Pacientes submetidos à ATQ podem iniciar a hidroterapia de 10 a 14 dias após a cirurgia, tendo como foco os exercícios com auxílio da flutuabilidade até três semanas seguidas do tratamento, quando a amplitude completa de movimentos pode ser motivada. Desta forma teremos como preferência nos exercícios o aumento da força nos músculos do quadril e perna (especialmente os extensores e abdutores do quadril, que são indispensáveis para caminhar), o aumento da amplitude de movimento (mantendo os limites prescritos) e corrigir as anormalidades da marcha, (CAMPION, 2000).

Bates e Hanson (1998), declaram que existem algumas limitações de movimentos para o paciente submetido à ATQ, envolvendo nenhuma adução que exceda a neutra, nenhuma flexão de quadril acima de $90^{\circ}$, nenhuma rotação externa e nenhum cruzamento das pernas.

Verificou que também há melhora nos quesitos funcionalidade, qualidade de vida e amplitude de movimento, em pacientes que foram submetidos à ATQ, quando realizadas 10 sessões de 60 minutos utilizando o aquecimento, a técnica Bad Ragaz com paciente em decúbito dorsal em flutuadores, exercícios de fortalecimento e treino de equilíbrio, (NUNES, 2010).

Segundo Bento et al. (2012), há um aumento de força muscular em indivíduos mais velhos saudáveis quando se comparado há um protocolo de exercícios aquáticos versus terrestres. A estratégia utilizada foi o gradual aumento da intensidade de exercício em cada quatro semanas, ampliando a área projetada dos membros inferiores e a velocidade de execução de movimentos, o que aumenta a resistência oferecida pela água.

A hidroterapia visa focalizar uma melhora na condição física do músculo, facilitação de músculo paréticos, resistência em casos de fraqueza muscular e desenvolvimento de força, facilitação da marcha e ortostatismo permitindo deambulação precoce, (BATES; HANSON, 1998).

\section{Análise de estudos da hidroterapia na artroplastia total do quadril}

Rahmann; Brauer; Nitz (2009), realizou um estudo que mostra os benefícios da hidroterapia na funcionalidade do quadril, ao avaliar o efeito da fisioterapia aquática sobre a 
recuperação da função, força e velocidade de marcha após ATQ. Foi iniciado o tratamento no $4^{\circ}$ dia do pós-operatório e a avaliação final realizada no $14^{\circ}$ dia. Foram inclusos 65 pacientes com idade média de 69,6 anos. Após dez dias de tratamento, a força abdutora do quadril foi significativamente maior após a função, a intervenção e a velocidade de marcha obtiveram melhora. Diante disto, concluíram que um programa de fisioterapia aquática hospitalar tem um efeito positivo na recuperação precoce do quadril após a cirurgia de substituição da articulação.

No estudo de caso realizado por Facci (2007), pacientes tratados com hidroterapia, obteve bons resultados em ganho de amplitude de movimento, além da evolução do quadro álgico e da qualidade de vida. Em vista disso, foi afirmado que estes obtidos foram suficientes, já que, o proposito fundamental do estudo foi melhora a capacidade funcional e, por resultado, a qualidade de vida destes pacientes.

Em estudos realizados por Wyatt et al. (2014), Wang et al. (2011), Wallis et al. (2014), Bressel et al. (2014) e Fisken et al. (2015), foram encontrados resultados para a funcionalidade e mobilidade que indicam que o exercício aquático com programas de duração de seis semanas ou mais e com 2 - 3 sessões semanais de 45 - 60 min pode ser eficaz melhorando a mobilidade e velocidade da marcha. A similaridade entre os movimentos realizados em tarefas diárias avaliada em testes funcionais e em exercícios aquáticos podem facilitar a transferência dos ganhos em exercícios.

Weigenfeld-Lahav et al. (2007), buscaram os efeitos de um programa de reabilitação por hidroterapia em doze atendimentos, após a substituição da articulação do quadril por condições artríticas. O tratamento foi realizado em 16 pacientes, com idade média de 56 anos, com pelo menos três meses de pós-operatório. Foram realizadas outras avaliações, onde os pacientes responderam ao questionário de qualidade de vida SF-36, seis semanas antes do início do tratamento, um dia antes e após as seis semanas que constituíam o programa. Concluíram que foram significativos os efeitos do tratamento proposto na qualidade de vida dos participantes, com destaque para os domínios da dor, limite por aspecto físico e saúde mental. Contudo, não foram todos os domínios que melhoraram significativamente. Os domínios do estado geral de vitalidade, saúde e aspectos sociais não apresentaram valores significativos. 
A hidrocinesioterapia, realizada em idosas sedentárias, no período de 24 a 28 sessões entre 55 e 60 minutos, com exercícios terapêuticos de alongamento, aquecimento, relaxamento e fortalecimento, mostrou resultados positivos em relação à melhora da força muscular e amplitude de movimento, (RIZZI, 2010 e CANDELORO, 2007). Isso ocorre por meio da resistência da água ao movimento, que pode ser incrementado o aumento da velocidade durante a execução dos exercícios, favorecendo assim o trabalho muscular, (GALIANO, 2013)

\section{Considerações finais}

Diante dos estudos analisados pode-se examinar que a hidroterapia é um benéfico e eficaz tratamento de pacientes de artroplastia total do quadril. A diminuição da dor foi o seu fundamental efeito terapêutico, acompanhada do ganho de flexibilidade constatada através do aumento da amplitude de movimento. A hidroterapia também se demonstrou eficaz para a melhora da capacidade funcional, da qualidade de vida, no quadro álgico, ganho de força muscular e redução de edema no membro operado nos pacientes submetidos à ATQ.

Após a realização da cirurgia de artroplastia total de quadril o paciente terá diversas limitações de mobilidade, devido ao grande espaço de imobilização, dor e fraqueza muscular.

A atividade fisioterapêutica no pós-operatório de artroplastia total de quadril é de relevante importância, pois viabiliza vantagens ao paciente tanto em termos da qualidade da recuperação como no mínimo tempo para atingi-la.

A hidroterapia é o local ideal para começar o atendimento de pacientes pós ATQ, pois aplica os efeitos fisiológicos da imersão combinados com o calor da água para tornar os movimentos mais fáceis, menos dolorosos, transformando a reabilitação mais eficaz e segura.

Diante disso podemos concluir que a hidroterapia seja um recurso indispensável para o tratamento pós-operatório de artroplastia total do quadril, pois acarreta diversos efeitos benéficos para a melhora de qualidade de vida do paciente.

\section{Referências}


ABNT NBR 14724. Associação Brasileira de Normas Técnicas: informação e documentação - Trabalhos acadêmicos - Apresentação. Rio de Janeiro: ABNT, 2005.

BATES, A.; HANSON, N. Exercícios aquáticos terapêuticos. São Paulo: Manole, 1998.

BENTO PCB, Pereira G, Ugrinowitsch C, Rodacki ALF. The effects of a water-based exercise program on strength an functionality of older adults. J Aging Phys Act. 2012.

BONVICINE, Cristiane. Proposta de intervenção fisioterapêutica precoce em pacientes submetidos à artroplastia total de quadril. 2006. 83 f. Dissertação (Mestrado em Ciências da Saúde) - Faculdade de Medicina de São José do Rio Preto. 2006. Disponível em: http://bdtd.famerp.br/bitstream/tede/6/1/cristianebonvicine_dissert.pdf. Acesso em: 19/04/2019.

BRESSEL E, Wing JE, Miller AI, Dolny DG. High-intensity interval training on an aquatic treadmill in adults with osteoarthritis: effect on pain, balance, function, and mobility. J Strength Cond Res. 2014.

CAMARGO JR, Luiz Lima de; DIONÍSIO, Felipe Nunes. Atuação da fisioterapia na lesão de labrum acetabular: revisão bibliográfica. Revista Ibirapuera, são Paulo, n. 12, jul./dez. 2016. Disponível em: <http://seer.unib.br/index.php/rev/article/view/94>. Acesso em: 05/05/2019.

CAMPION, Margharet Reid. Hidroterapia: princípios e prática. São Paulo: Manole, 2000.

CANDELORO, J.M.; CAROMANO, F.A. Efeito de um programa de hidroterapia na flexibilidade e na força muscular de idosas. Rev. Bras. Fisioter, São Carlos, v.11, n.4, jul./ago. 2007.

CARREGARO Rodrigo Luiz; Toledo Aline Martins de; Efeitos Fisiológicos e evidências cientificas da eficácia da fisioterapia aquática. Revista a Movimenta. vol. 1, n. 1, 2008.

DIDOMENICO, Letícia; PAES, Mayra Battistini. Fisioterapia na artroplastia de quadril: relato de caso. 2010. Monografia (Graduação em fisioterapia) - Centro Universitário Católico Salesiano Auxilium - UNISALESIANO. Lins - SP. 2010. Disponível em: <http://www.unisalesiano.edu.br/biblioteca/monografias/51834.pdf>. Acesso em: 15/05/2019.

DUTTON, Mark. Fisioterapia Ortopédica: Exame, Avaliação e Intervenção. 1 ed. Porto Alegre - RS: Artmed, 2006. 
DUTTON, Mark. Fisioterapia Ortopédica: Exame, Avaliação e Intervenção. 2. ed. Porto Alegre - RS: Artmed, 2010. Disponível em: </integrada.minhabiblioteca.com.br/\#/books/ 9788536323718/cfi/0!/4/2@100:0.00>. Acesso em: 18/05/2019.

FACCI, Ligia Maria; Marquetti Renata; Coelho Kelley Cristina; Fisioterapia aquática no tratamento da osteoartrose de joelho: série de casos. Fisioterapia em Movimento, Curitiba, v. 20, n. 1, jan./mar., 2007.

FERREIRA, L.R.F; PESTANA, P.R; OLIVEIRA, J; FERRARI, R.A.M. Efeitos da reabilitação aquática na sitomatologia e qualidade de vida de portadoras de artrite reumatóide. Fisioterapia e Pesquisa, São Paulo, v.15, n.2, abr./jun. 2008.

FISKEN AL, Waters DL, Hing WA, Steele M, Keogh JW. Comparative effects of 2 aqua exercise programs on physical function, balance, and perceived quality of life in older adults with osteoarthritis. J Geriatr Phys Ther. 2015.

GALEA, M. et al. A targeted home-and centerbased exercise program for people after total Hip Replacement: A Randomized Clinical Trial. Arch. Phys. Med. Rehabil. v. 89, 2008 .

GALIANO, P; SANTOS, R.V. Efeitos da hidroterapia no portador de atrogripose múltipla congênita: um relato de caso. Fisi Senectus, Unochapecó, v.1, n.2, jul./dez. 2013.

GANN, Nancy. Ortopedia Guia de Consulta Rápida para Fisioterapia. Rio de Janeiro RJ: Guanabara Koogan. 2005.

GIMENES, Rafaela Okano et al. Análise crítica de ensaios clínicos aleatórios sobre fisioterapia aquática para pacientes neurológicos. Revista neurociências. v.13, n. 1, jan./mar 2005. Disponível em: <http://www.unifesp.br/dneuro/neurociencias/Neurociencias \%20V13\%20N1_28_07_05.pdf>. Acesso em: 20/01/2019.

HERBERT, Sizinio et al., Ortopedia e Traumatologia: Princípios e Prática. $4^{\mathrm{a}}$ ed. Porto Alegre. Editora: Artmed. 2009. Disponível em: https://integrada.minhabiblioteca.com. br/\#/books/9788536318509/cfi/0!/4/2@100:0.00. Acesso em: 20/04/2019.

KISNER, Carolyn; COLBY, Lynn Allen. Exercícios terapêuticos: fundamentos e técnicas. 5 ed. Barueri - SP: Manole, 2009.

KISNER, Carolyn; COLBY, Lynn Allen. Exercício Terapêutico: fundamentos e técnicas. 6. ed. São Paulo: Manole, 2016. 
LIPPERT, Lynn. Cinesiologia Clinica e Anatomia. 5.ed. Rio de Janeiro. RJ: Guanabara Koogan: 2013. Disponível em: <https://integrada.minhabiblioteca.com.br/\#/books/978-85277-2235-3/cfi/0!/4/2@100:0.00>. Acesso em: 28/04/2019.

MATOS, Denise Regina. Qualidade de vida e reabilitação: casos cirúrgicos de artroplastia total do quadril, 2006. Dissertação (Mestrado em Psicologia) - Instituto de Psicologia. Universidade de Brasília. 2006. Disponível em: <http://pesquisa.bvs.br/brasil/ resource/pt/pte-35516 >. Acesso em: 13/05/2019.

MEEREIS, E.C.W; FAVRETTO, C; SOUZA, J; GONÇALVES, M.P; MOTA, C.B. Influência da hidrocinesioterapia no equilíbrio postural de idosas institucionalizadas. Motriz, Rio Claro, v.19, n.2, abr./jun. 2013.

MENEGHETTI, C.H.Z; BASQUEIRA. C; FIORAMONTE. C; JUNIOR. L.C.F. Influência da fisioterapia aquática no controle de tronco na síndrome de pusher: relato de caso. Fisioterapia e Pesquisa, São Paulo, v.16 n. 3, jul./set. 2009.

MORAES, Clarisse Niero. Efeito do método dos anéis do Bad Ragaz na reabilitação de pacientes submetidos à artroplastia total de quadril. (Monografia de Fisioterapia) Universidade do Sul de Santa Catarina. 2005. Disponível em:<http://www.fisiotb.unisul.br/Tccs/ClarissaNieiro/tcc.pdf>. Acesso em: 02/03/2019.

NUNES, Guilherme Silva; KOERICH, Micheline Henrique Araújo da Luz; MENEZES, Fábio Sprada de. Abordagem Hidroterapêutica em Pacientes Submetidos à Artroplastia Total de Quadril - Um Estudo Multicaso. Arquivos de Ciências da Saúde. UNIPAR, Umuarama, v. 14, n. 2, maio/ago. 2010. Disponível em: <http://revistas.unipar.br/ index.php/saude/article/viewFile/3417/2319>. Acesso em:10/04/2019.

OLIVEIRA VG; SOUSA DPM, Hidroterapia como tratamento fisioterapêutico de osteoartrite de quadril utilizando o método Bad Ragaz, 2018. Disponível em: http://portalbiocursos.com.br/ohs/data/docs/33/175_Hidroterapia_como_tratamento_fisioterap Yutico_de_osteoartrite_de.pdf. Acesso em:10/04/2019.

PEREIRA Bruna Rogeliane, Rodrigues et al. Artroplastia do quadril: prevenção de infecção do sítio cirúrgico. Revista SOBECC. São Paulo. 19(4). out./dez., 2014. Disponível em: <http://sobecc.org.br/arquivos/artigos/2015/pdfs/v19n4/SOBECC_v19n4_181-187.pdf>. Acesso em: 10/05/2019.

PRENTICE, William. Fisioterapia na Prática Esportiva. Uma abordagem baseada em competências. 14 ${ }^{\mathrm{a}}$ Ed. Porto Alegre - RS: Artmed. 2012. 
RAHMANN, A. E.; BRAUER, S. G.; NITZ, J. C. A specific inpatient aquatic physiotherapy program improves strength after total hip or knee replacement surgery: a randomized controlled trial. Arch. Phys. Med. Rehabil. v. 90, 2009.

RIZZI, P.R.S; LEAL, R.N; VENDRUSCULO, A.P. Efeito da hidrocinesioterapia na força muscular e na flexibilidade em idosas sedentárias. Fisioter. Mov, Curitiba, v. 23, n. 4, out./dez. 2010.

ROSA, Tabada Samantha et all. Fisioterapia aquática como prevenção de quedas na terceira idade: revisão de literatura. Cinergis 2013. http://online.unisc.br/seer/Index .php/cinergis. Acesso em 19/05/2019.

SCHWARTSMANN, Carlos Roberto et al. Novas superfícies em artroplastia total do quadril. Revista Brasileira de ortopedia. V. 47, n.2. 2012. Disponível em:<http://www.scielo. br/scielo.php?script=sci_arttext\&pid=S0102-36162012000200002 >. Acesso em: 27/02/2019. SILVA, K.M.O.M; TUCANO, S.J.P; KUMPEL, C; CASTRO, A.A.M; PORTO, E.F. Efeito da hidrocinesioterapia sobre qualidade de vida, capacidade funcional e qualidade do sono em pacientes com fibromialgia. Ver Bras Reumatol, v.52, n.6, nov./dez. 2012.

SILVA, Paulo et al. Artroplastia total de quadris displásicos luxados com reconstrução acetabular e encurtamento femural. Revista brasileira de ortopedia. v.49, n.1, 2014. Disponível em: <http://www.scielo.br/scielo.php?pid=S0102-36162014000100069 \&script=sci_abstract\&tlng=p>. Acesso em: 25/02/2019.

SKINNER, Harry; Mc MAHON, Patrick. Current ortopedia: diagnóstico e tratamento. 5 ed. Artmed. Porto Alegre - RS. 2015. Disponível em: <https://integrada.minhabiblioteca.com. br/\#/books/9788580554366/cfi/3 >. Acesso em: 15/05/2019.

SOARES, Nayara et all. Técnicas terapêuticas da hidroterapia aplicadas na reabilitação de pacientes com artrite reumatoide juvenil: revisão de literatura. EFDeportes.com, Revista Digital. Buenos Aires, Año 19, No 197, Octubre de 2014. http://www.efdeportes.com.

STRÖM, H. et al. The effect of early weight bearing on migration pattern of the uncemented CLS stem in total hip arthroplasty. J. Arthroplasty, v. 22, n. 8, 2007.

TORTORA, Gerard; DERRICKSON, Bryan. Corpo Humano - fundamentos de Anatomia e Fisiologia. 10 ed. Artmed. São Paulo - SP. 2015.

VIEIRA JR, OLIVEIRA MA, LUZES R. Efeitos da hidroterapia em pacientes idosos com osteoartrose de joelho. Alumni-Revista Discente da UNIABEU, 2017. Disponível em: http://revista.uniabeu.edu.br/index.php/alu/article/view/2179/1774. Acesso em: 25/02/2019. 
WAGENMAKERS, R. et al. Habitual physical activity behavior of patients after primary total hip arthroplasty. Phys. Ther. v. 88, n. 9, 2008.

WALLIS JA, Webster KE, Levinger P, Fong C, Taylor NF. A pre-operative group rehabilitation programme provided limited benefit for people with severe hip and knee osteoarthritis. Disabil Rehabil. 2014.

WANG TJ, Lee SC, Liang SY, Tung HH, Wu SF V, Lin YP. Comparing the efficacy of aquatic exercises and land-based exercises for patients with knee osteoarthritis. $\mathrm{J}$ Clin Nurs. 2011.

WEIGENFELD-LAHAV, I. et al. Physical and psychological effects of aquatic therapy in participants after hip-joint replacement: a pilot study. Int. J. Aquat. Res. Educ. v. 1, 2007.

WYATT yatt M, Hooper G, Frampton C, Rothwell A. Survival outcomes of cemented compared to uncemented stems in primary total hip replacement. World J Orthop. 2014. 